\title{
Truncated co-seismic geoid and gravity changes in the domain of spherical harmonic degree
}

\author{
Wenke Sun and Shuhei Okubo \\ Earthquake Research Institute, University of Tokyo, Yayoi 1-1-1, Bunkyo-ku, Tokyo 113-0032, Japan
}

(Received February 9, 2004; Revised June 8, 2004; Accepted July 26, 2004)

\begin{abstract}
A concept of truncated geoid and gravity changes is proposed in this study and corresponding truncated expressions are presented for investigating co-seismic deformations. Numerical investigations are carried out to observe whether or not co-seismic geoid and gravity changes are detectable by gravity satellite missions. Results of an individual harmonic degree or a summation to interested degrees are compared with the expected errors of the gravity missions, assuming a seismic source equivalent to the fault size of the Alaska earthquake $\left(1964, m_{w}=9.2\right)$. Corresponding co-seismic deformations indicate that both the gravity and geoid changes are about two orders larger than the precision of GRACE. Based on these results, the minimum magnitudes of earthquakes detectable by GRACE are derived. The conclusion is that co-seismic deformations for an earthquake with a seismic magnitude above $m=7.5$ (for the tensile sources) and $m=9.0$ (for the shear sources) are expected to be detected by GRACE. Finally, a case study is made on the 2002 Alaska earthquake $(m=7.9)$. Results show that the co-seismic geoid and gravity changes are at or below the error level of GRACE, and are difficult to detect.
\end{abstract}

Key words: Co-seismic deformation, geoid, gravity, gravity mission, dislocation, earthquake.

\section{Introduction}

Dedicated satellite missions, such as the Challenging Mini-Satellite Payload (CHAMP), the Gravity Recovery and Climate Experiment (GRACE), and the Gravity Field and Steady-state Ocean Circulation Explorer (GOCE) provide an estimate of constant and time-variable components of the earth's gravity field with unprecedented accuracy, global, and high-resolution. It can be expected that most time change problems in geosciences can be detected and well studied in the near future. A potential application of the satellite gravity missions, e.g., GRACE, is that they provide us with powerful means to detect co-seismic gravity and geoid changes. A case study of the 1964 Alaska earthquake (Sun and Okubo, 1998) indicated that gravity change could be detected on the earth surface. However, it is questionable whether such gravity and geoid height changes can be detected or not by modern space techniques like altimetry and gravity missions. This question cannot be answered simply by results of surface gravity changes as a result of the spatial resolution limit of the gravity satellite missions. This requires a corresponding investigation for individual or group wavelength. That is, theoretical work and simulation on a truncated co-seismic deformation is necessary to calculate co-seismic deformations with a truncation of the harmonic degrees. Consequently, the contribution from the high-degree component can be removed. These calculations and simulations can be achieved using the dislocation theory, e.g., by Sun and Okubo (1993), for a spherical earth because it is expressed in the form of spherical harmonics. Gross and

Copy right (c) The Society of Geomagnetism and Earth, Planetary and Space Sciences (SGEPSS); The Seismological Society of Japan; The Volcanological Society of Japan; The Geodetic Society of Japan; The Japanese Society for Planetary Sciences; TERRAPUB.
Chao (2001) investigated this problem using normal mode technique. Comparing the degree amplitude spectra of some earthquakes with the expected GRACE errors, they found that the co-seismic effects of great earthquakes such as the 1960 Chilean or 1964 Alaska events can cause global gravitational field changes that are sufficiently large to be detected by GRACE.

Sun and Okubo (2004) derived theoretical formulations of co-seismic geoid and gravity changes and their degree variances, expressed by dislocation Love numbers. They investigated co-seismic geoid and gravity changes by observing the distribution of their degree variances in comparison to the expected sensitivity of satellite gravity missions. Results for co-seismic deformations for large earthquakes are discussed with respect to their detectability. Their study offered an identical conclusion to that of Gross and Chao (2001) using the normal mode scheme.

However, from a new way, this study presents a concept of truncated geoid and gravity changes and corresponding expressions. Assuming a large earthquake equivalent to the 1964 Alaska earthquake $\left(m_{w}=9.2\right)$, we investigate spatial distributions of the co-seismic geoid and gravity changes for each individual and group wavelength within the spatial resolution of the gravity missions. The results are used to compare with the expected error of GRACE, so that the minimum seismic magnitude to be detected by GRACE is derived. Results lead to the same conclusion as Gross and Chao (2001) and Sun and Okubo (2004). The theory and results in this paper are expected to have wider applications in the future. A case study is also made on the 2002 Alaska earthquake $(m=7.9)$. Note that for temporal co-seismic changes, a more realistic earth model, such as a visco-elastic model should be considered, as did by Pollitz (1997) and 


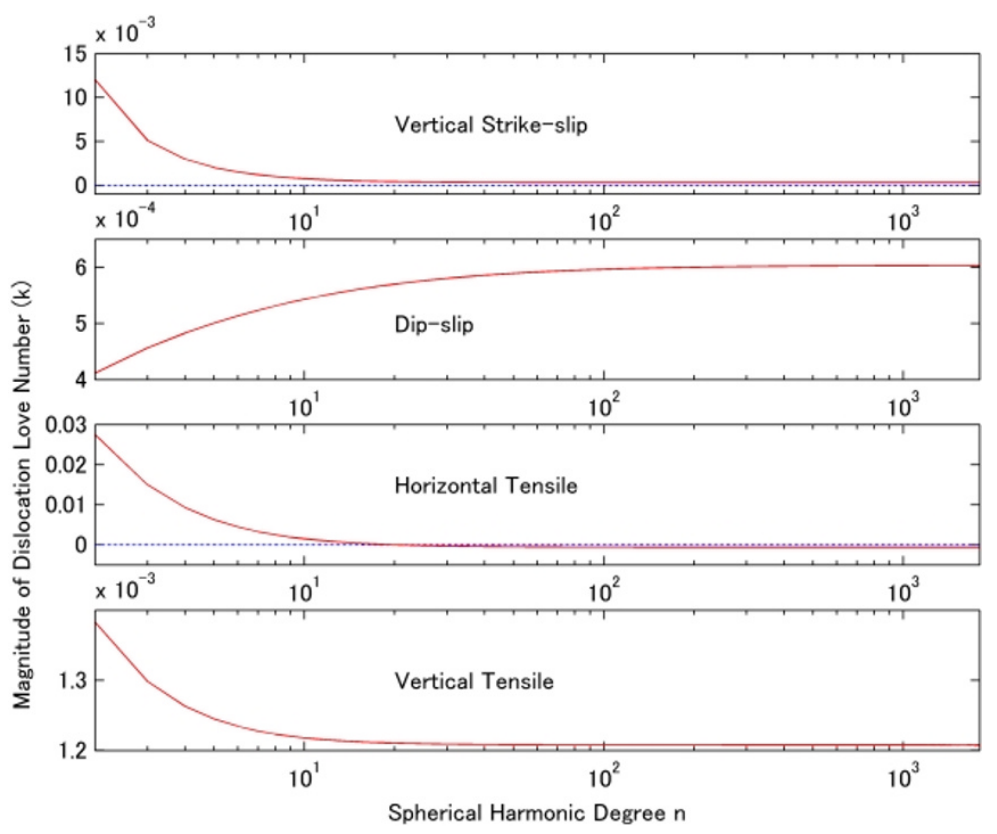

Fig. 1. Normalized dislocation Love numbers $k_{n m}^{i j}$ of four types of seismic sources at a depth of $32 \mathrm{~km}$.

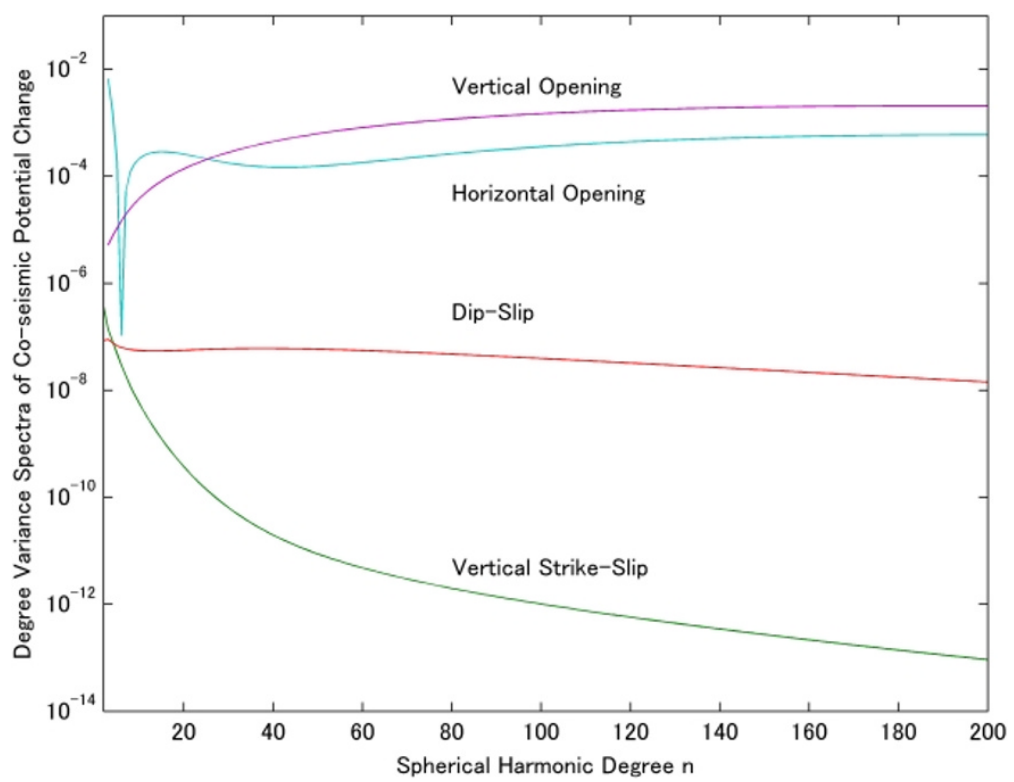

Fig. 2. Degree variance spectra (power spectral density) of co-seismic potential change for four types of dislocation sources at a depth of $32 \mathrm{~km}$.

Wang (1999)

\section{Dislocation Love Numbers and Co-seismic Geoid and Gravity Changes}

According to the quasi-static dislocation theory, for a point dislocation located on the polar axis of a compressible and self-gravitating spherical earth, co-seismic geoid and gravity changes at an observing point $(a, \theta, \varphi)$ on the deformed earth surface can be expressed as a combination of dislocation Love numbers $h_{n m}^{i j}$ (concerning the vertical displacement) and $k_{n m}^{i j}$ (related to the gravitational potential change) (Sun and Okubo, 1993). However, for the gravity measurements by satellites, the term due to the vertical displacement vanishes, so that the corresponding co-seismic geoid and gravity changes are:

$$
\begin{gathered}
\zeta^{i j}(a, \theta, \varphi)=\sum_{n, m} k_{n m}^{i j} Y_{n}^{m}(\theta, \varphi) \cdot v_{i} n_{j} \frac{U d S}{a^{2}} \\
\delta g^{i j}(a, \theta, \varphi)=\sum_{n, m}(n+1) k_{n m}^{i j} Y_{n}^{m}(\theta, \varphi) \cdot v_{i} n_{j} \frac{g_{0} U d S}{a^{3}} .
\end{gathered}
$$

The dislocation Love numbers are functions of the spherical harmonic degree, order, source depth, and type. Components of the slip vector and its normal on the infinitesimal fault area $d S$ are $v_{i}$ and $n_{j}$, with total dislocation $U$. Gravity on the earth surface is $g_{0}$; a is the radius of the Earth and $Y_{n}^{m}(\theta, \varphi)$ is the spherical harmonic function of degree $n$ and order $m$. The two factors $U d S / a^{2}$ and $g_{0} U d S / a^{3}$, called dislocation 
factors, define the earthquake magnitude and give the unit of geoid and gravity changes.

A combination of the three slip and normal components implies that there are nine total solutions for all possible sources. However, if the earth model is spherically symmetric and isotropic, there are only four independent solutions. A deformation caused by an arbitrary source can be obtained by a proper combination of the four types of independent sources. In this study, we choose the following four independent solutions: $i j=12,32,22$, and 33. They represent strike-slip, dip-slip, horizontal tensile and vertical tensile, respectively. Note that the components of $i j=22$ include two parts: $m=0$ and 2; we here calculate and discuss only the deformations of $m=0$ : the computation of $m=2$ can be derived easily from the component of $i j=12$. Details can be found in Sun and Okubo (1993) or Sun et al. (1996).

The dislocation Love numbers $k_{n m}^{i j}$ can be numerically obtained for a spherically symmetric earth model (Sun and Okubo, 1993), such as the 1066A (Gilbert and Dziewonski, 1975) or the PREM (Dziewonski and Anderson, 1981). Then the gravity and geoid changes can be calculated by the above summations in (1) and (2). Figure 1 gives numerical results of the dislocation Love numbers $k_{n m}^{i j}$ of the four types of seismic sources at a depth of $32 \mathrm{~km}$ with the $1066 \mathrm{~A}$ model as a function of the spherical harmonic degree $n$ up to 2000 . The dislocation Love numbers vary rather smoothly as $n$ increases. Once a dislocation source or earthquake parameter is provided, co-seismic deformations can be easily calculated using these Love numbers.

It is difficult to observe behaviors in the high-degree portion because the dislocation Love numbers in Fig. 1 decay quickly as $n$ increases and even change sign. To compare the relative contributions of each source, we calculate the spectrum power (root square of each individual harmonic degree) of these dislocation Love numbers for four types of sources. Figure 2 depicts the degree variation spectra of co-seismic potential changes to a harmonic degree of 200 . The two tensile sources apparently have stronger power than the two shear sources. This means that co-seismic deformations for tensile sources are expected to be larger than those of the shear sources. However, if spherical harmonic order $m$ is considered, i.e., they were 0,1 , and 2 , respectively; the real co-seismic responses would behave more closely to some degree.

In a previous study (Sun and Okubo, 2004), it was proved that the dislocation Love numbers in Fig. 2 are nothing but the Stokes coefficients of the co-seismic deformations. Since the source is chosen at the polar axis, and due to the symmetric property of the source functions, the angular order $m$ damps except $m=0,1$ and 2. It means that the coefficients for all other angular orders are zero. They can be directly used to compare with the expected error of the GRACE measurement to observe the detectability of co-seismic deformations from space. In the following sections, however, some expressions are presented for practical applications of the quasi-static dislocation theory to compute truncated coseismic deformations. At the same time, the same conclusion as presented in Sun and Okubo (2004) can also be reconfirmed.

\section{Co-seismic Geoid and Gravity Changes by Har- monic Degrees}

In this section, there are two aims: 1) to derive expressions for computing co-seismic deformations of each individual harmonic degree for later application; 2) through numerical investigation of the spatial distributions of co-seismic deformations for each individual harmonic degree to find out the minimum seismic amplitude to be detected by space techniques. Actually the latter is a parallel way to the previous study (Sun and Okubo, 2004), and gives the same conclusion. Since the satellite gravity missions provide geoid and gravity measurements in the form of spherical harmonic coefficients with limited spatial resolution, we should study the co-seismic deformations correspondingly in the spectrum domain. So that it is straightforward to investigate whether co-seismic deformations are detectable by the satellite gravity missions, e.g., GRACE. For this purpose, the expressions for each $n$ and $m$, and a fixed co-latitude $\theta=\Theta$, can be easily derived from Eqs. (1) and (2):

$$
\begin{gathered}
\zeta^{i j}(n ; a, \Theta, \varphi)=k_{n m}^{i j} Y_{n}^{m}(\Theta, \varphi) \cdot v_{i} n_{j} \frac{U d S}{a^{2}} \\
\delta g^{i j}(n ; a, \Theta, \varphi)=(n+1) k_{n m}^{i j} Y_{n}^{m}(\Theta, \varphi) \cdot v_{i} n_{j} \frac{g_{0} U d S}{a^{3}} .
\end{gathered}
$$

In a practical calculation, parameters such as source type $i j$ and harmonic order $m$ are determined according to one of the four source types. For example, for a vertical strike-slip fault, $i j=12, m=2$ : the expression of the co-seismic geoid change becomes

$$
\zeta^{12}(n ; a, \Theta, \varphi)=2 \sin 2 \varphi k_{n 2}^{12} P_{n}^{2}(\cos \Theta) \cdot \frac{U d S}{a^{2}} .
$$

Other components can be expressed similarly. Note that the co-seismic deformations in Eqs. (3) and (4) should be calculated not only for co-latitude $\theta$, but also for longitude $\varphi$. However, only the maximum change is needed to determine whether GRACE can detect the signal of the co-seismic deformations. So let us consider only cases of $\sin 2 \varphi=1$ for the vertical strike-slip source, and $\sin \varphi=1$ for the dip-slip source, assuming a great fault equivalent to that of the Alaska earthquake $\left(1964, m_{w}=9.2\right)$ with parameters (Savage and Hastie, 1966) length $=600 \mathrm{~km}$, width $=200 \mathrm{~km}$, and dislocation $=10 \mathrm{~m}$. Thereby, the dislocation factors in (3)-(4) yield $U d S / a^{2}=2.956 \mathrm{~cm}$ for geoid change and $g_{0} U d S / a^{3}=4.556 \mu$ gal for gravity change. To investigate all cases of seismic sources, the same magnitude of the fault size for the four independent sources is assumed at a depth of $32 \mathrm{~km}$ buried in the 1066A earth model. Once the results for the Alaska earthquake case are obtained, a co-seismic deformation caused by an arbitrary earthquake can be easily estimated.

Using the dislocation Love numbers in Fig. 1, we compute co-seismic geoid and gravity changes using Eqs. (3) and (4), for several epicentral distances. It should be pointed out that, ideally, the co-seismic deformations should be investigated for the whole epicentral distances on the earth surface. However, it should be enough to consider some typical distances provided they present the maximum changes. Therefore, we only consider the following four epicentral distances: $0.01^{\circ}$, 

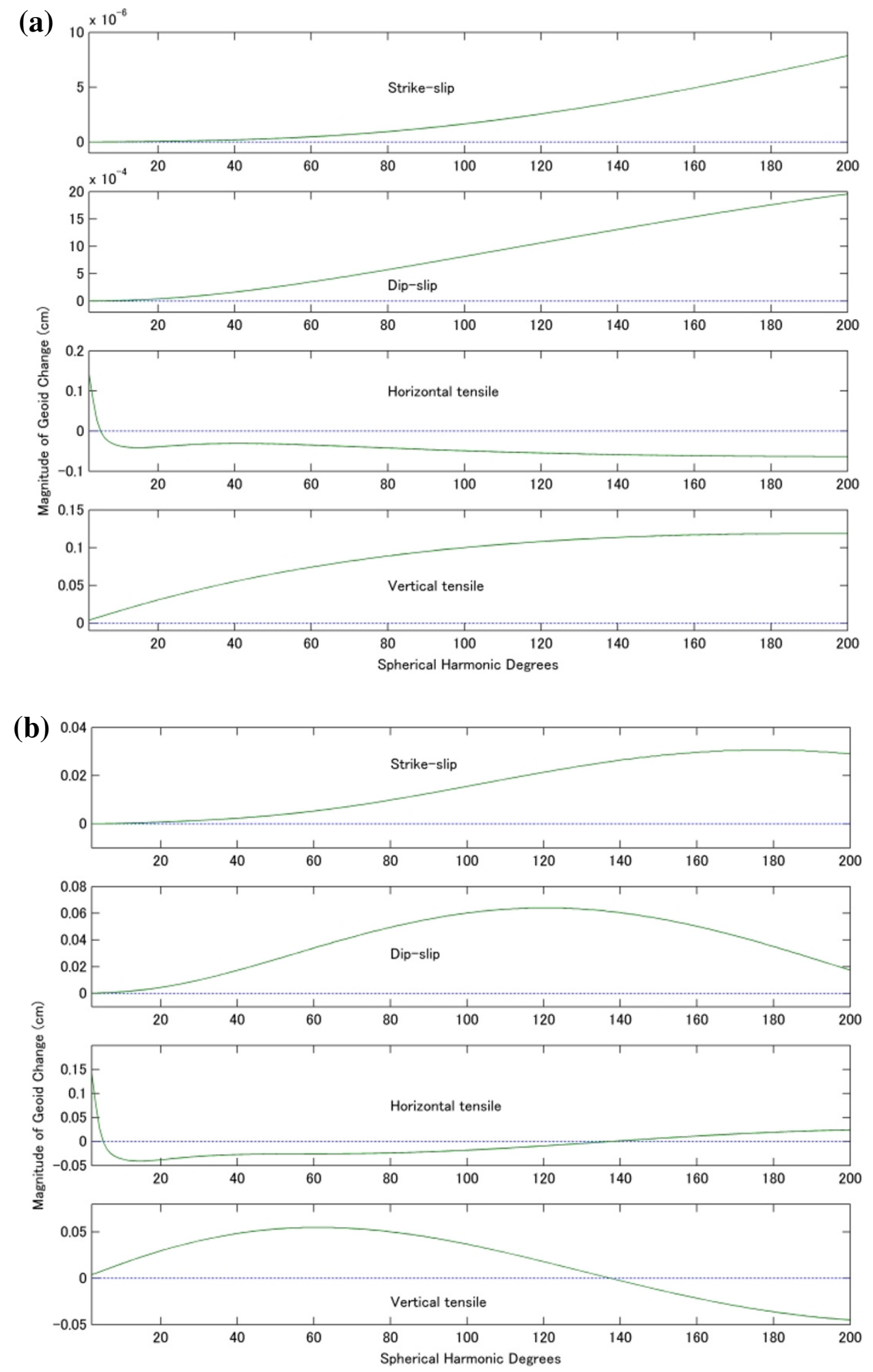

Fig. 3. Co-seismic geoid changes at four epicentral distances caused by four types of seismic sources at a depth of $32 \mathrm{~km}$ : (a) $0.01^{\circ}$, (b) $1^{\circ}$, (c) $10^{\circ}$, and (d) $20^{\circ}$.

$1^{\circ}, 10^{\circ}$ and $20^{\circ}$. Results of the co-seismic geoid changes are plotted in Fig. 3. The x-axis gives the harmonic degree $n$ to 200; the y-axis shows the contribution of each harmonic degree to the co-seismic geoid change with a unit of centimeters. Subplots in Fig. 3 present the four independent solutions. Contributions from each harmonic degree are different for different angular distances. For example, for an angular distance of $1^{\circ}$, the maximum co-seismic geoid change of a strike-slip source is about $0.04 \mathrm{~mm}$ at $n=180$, whereas the maximum geoid change of a dip-slip fault is about $0.6 \mathrm{~mm}$, which corresponds to a harmonic degree of 120 . The maximum geoid change of a horizontal extension is about $1 \mathrm{~mm}$ at harmonic degree of 2, whereas the maximum geoid change of the vertical extension reaches $0.6 \mathrm{~mm}$ at $n=60$. The con- 
(c)
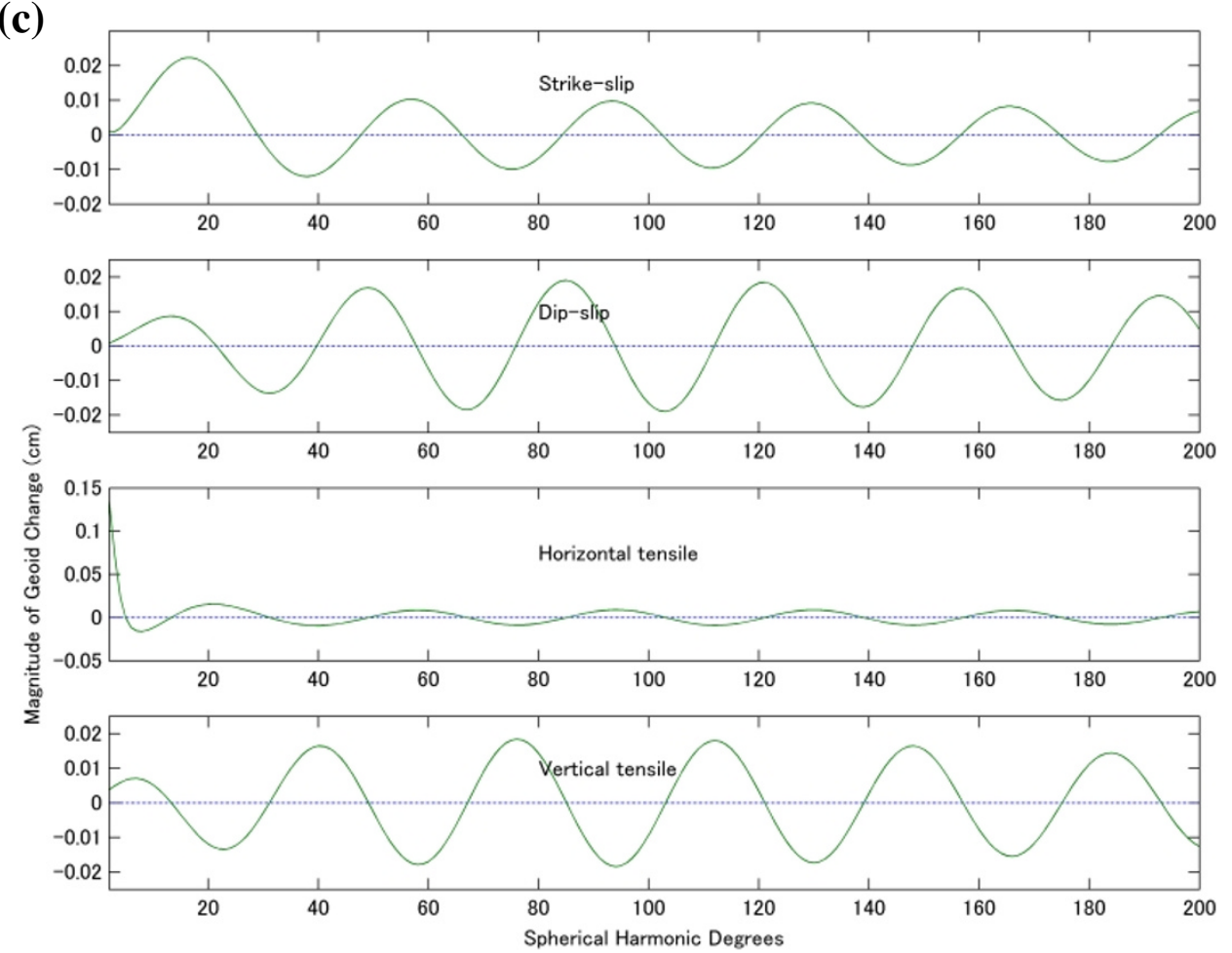

(d)
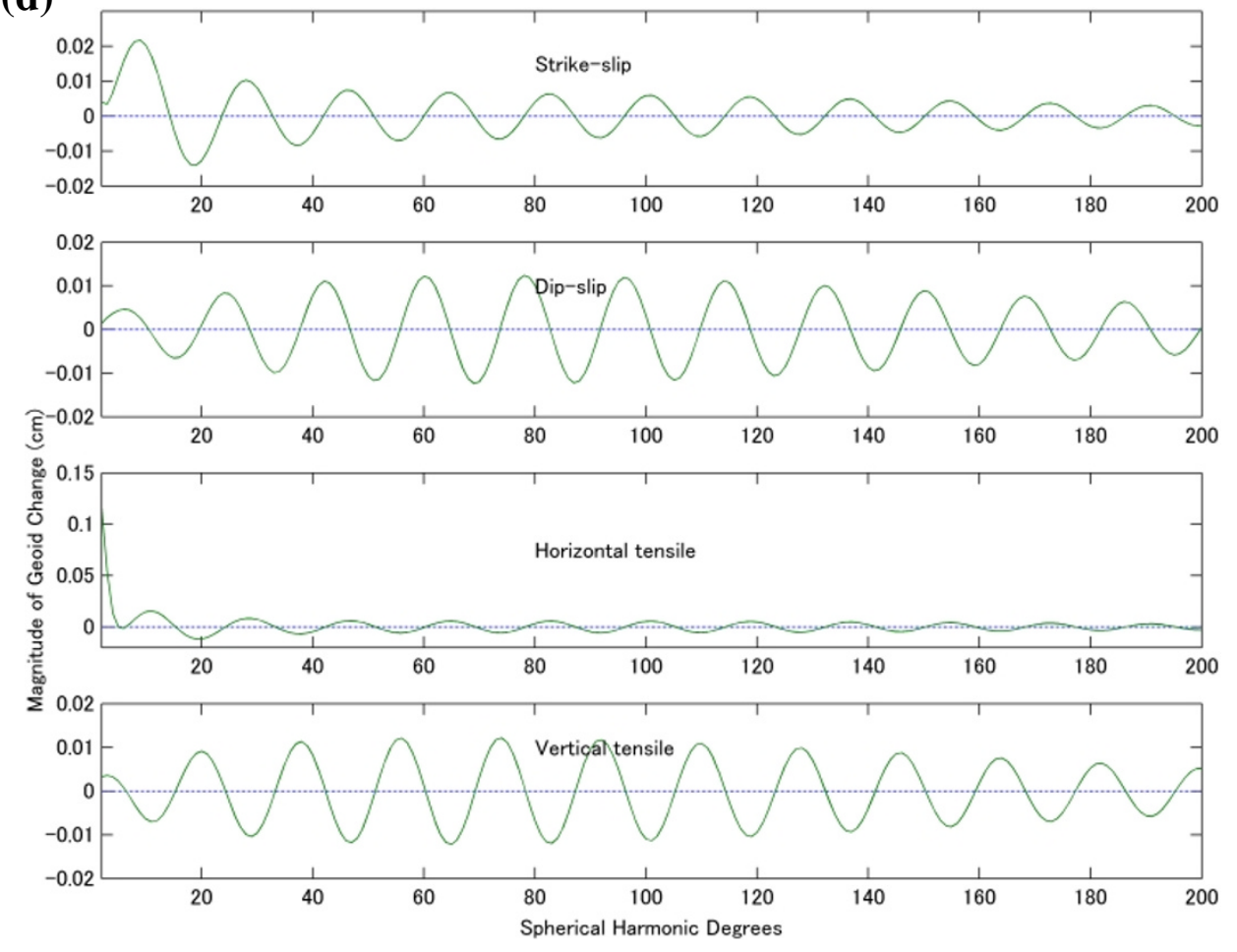

Fig. 3. (continued).

tribution of harmonic degree to geoid change at $10^{\circ}$ (and $20^{\circ}$ ) shows different behavior: it appears that wavelength varies as $n$ increases.

Since co-seismic gravity changes show similar results as the geoid changes, they are not plotted here. The results show that the gravity change magnitude is several to tens of microgals. Generally, co-seismic deformations appear to be very complicated because they are functions of dislocation types, source depth, and observation positions. For convenience, we summarize these results in the following tables with only the maximum changes, which we are interested in.

Table 1 lists the maximum co-seismic geoid changes in absolute value for different harmonic degrees $n$. The four columns show results for four different angular distances; the 
Table 1. Maximum co-seismic geoid changes (absolute value; $\mathrm{mm}$ ) at different epicentral distances.

\begin{tabular}{ccccc}
\hline $\begin{array}{c}\text { Source } \\
\text { type } i j\end{array}$ & $0.01^{\circ}$ & $1^{\circ}$ & $10^{\circ}$ & $20^{\circ}$ \\
\hline 12 & 0.0001 & 0.3 & 0.2 & 0.2 \\
& $(n>200)$ & $(n>100)$ & $(n=18)$ & $(8<n<20)$ \\
32 & 0.002 & 0.7 & 0.2 & 0.2 \\
& $(n>200)$ & $(n=120)$ & $(30<n<180)$ & $(60<n<120)$ \\
22 & 1.5 & 1.1 & 1.0 & 1.0 \\
33 & $(n=2)$ & $(n=2)$ & $(n=2)$ & $(n=2)$ \\
& $1.2(n>140)$ & 0.6 & 0.2 & 0.15 \\
& $>0.5(n>30)$ & $(n=60)$ & $(40<n<120)$ & $(50<n<120)$ \\
\hline
\end{tabular}

Table 2. Anticipated geoid height errors derived from 30 day GRACE data.

\begin{tabular}{cc}
\hline $\begin{array}{c}\text { Harmonic } \\
\text { degree }\end{array}$ & $\begin{array}{c}\text { Geoid height error } \\
(\mathrm{mm})(\text { per } \mathrm{n})\end{array}$ \\
\hline$n=2$ & $<0.10$ \\
$3 \leq n \leq 10$ & $<0.01$ \\
$10 \leq n \leq 70$ & $<0.15$ \\
$70 \leq n \leq 100$ & $<1.50$ \\
$100 \leq n \leq 150$ & $<65.0$ \\
\hline
\end{tabular}

Table 3. Minimum magnitude of earthquakes expected to be detected by gravity missions.

\begin{tabular}{ccc}
\hline $\begin{array}{c}\text { Source } \\
\text { type } i j\end{array}$ & GRACE & $\begin{array}{c}\text { GRACE } \\
\text { follow-on }\end{array}$ \\
\hline 12 & $m=9.0$ & $m=7.5$ \\
32 & $m=9.0$ & $m=7.5$ \\
22 & $m=7.5$ & $m=6.0$ \\
33 & $m=7.5$ & $m=6.0$ \\
\hline
\end{tabular}

four rows show results from the four types of sources. The unit is millimeters. The case of vertical strike-slip indicates that the geoid changes $0.0001 \mathrm{~mm}$ at $0.01^{\circ}$ when $n>200$; whereas it changes $0.3 \mathrm{~mm}$ at $1^{\circ}$ when $n>100$. Generally, the co-seismic geoid changes of the two tensile sources are larger than those of the shear sources, as discussed in their power spectra comparison (Section 2). On the other hand, according to simulation results for GRACE, Table 2 lists the anticipated geoid height errors derived from 30 days of GRACE data. For example, it shows that the geoid height error is less than $0.01 \mathrm{~mm}$ for a harmonic degree $n$ between three and ten. Then, a comparison between Tables 1 and 2 indicates that the geoid height changes caused by an earthquake as large as the Alaska earthquake (1964) are much larger than the errors of GRACE (see Table 1): they are even two orders larger than those errors. This fact implies that the co-seismic geoid changes are certainly detectable by gravity missions.

According to the above results and discussions, we may easily derive the minimum magnitudes of earthquakes which are expected to be detected by the gravity missions. Table 3 lists the results. They show that, if an earthquake is as large as the magnitude of $m=9$ (for source types 12 and 32) or $m=7.5$ (for source types 22 and 33), the corresponding co-seismic deformations are expected to be detected by GRACE. Whereas the last column in Table 3 shows that if an earthquake is as large as a magnitude of $m=7.5$ (for shear sources) or $m=6.0$ (for tensile sources), the co-seismic geoid and gravity changes are expected to be detected by GRACE follow-on, if it is two orders better in accuracy than GRACE (Watkins et al., 2000; NRC, 1997).

Note that in this section, we show the truncated geoid changes induced by the 1996 Alaska earthquake $(m=9.2)$ with cut-off degree $n=200$ which corresponds the wavelength of about $100 \mathrm{~km}$, smaller than the fault length of the earthquake. However, this case can be treated as a point source based on the following reasons: basically this is an estimation of the GRACE detectability and it should cover any cases, such as a small geometrical fault size but a big dislocation. In other words, only the same seismic moment (or magnitude) but for four independent sources are considered. Readers can take this investigation for a general case. On the other hand, the above results indicate that the relative large co-seismic deformations mainly appear in the lower harmonic degrees. Therefore, it can be expected that the conclusion in Table 3 would be almost the same even though a limited fault is considered.

\section{Co-seismic Geoid and Gravity Changes of Each Harmonic Degree for Different Epicentral Dis- tance}

Next, we investigate co-seismic geoid and gravity changes of each harmonic degree for different epicentral distance. In this case, the spherical harmonic degree is fixed ( $n=$ $N$ ) and epicentral distance changes, so that corresponding expressions of the co-seismic geoid and gravity changes can be written as

$$
\begin{gathered}
\zeta^{i j}(a, \theta, \varphi)=k_{N m}^{i j} Y_{N}^{m}(\theta, \varphi) \cdot v_{i} n_{j} \frac{U d S}{a^{2}} \\
\delta g^{i j}(a, \theta, \varphi)=(N+1) k_{N m}^{i j} Y_{N}^{m}(\theta, \varphi) \cdot v_{i} n_{j} \frac{g_{0} U d S}{a^{3}} .
\end{gathered}
$$

For example, for harmonic degree of $n=20$, the practical formula for calculating co-seismic geoid changes caused by a vertical strike-slip fault is

$$
\zeta^{12}(a, \theta, \varphi)=2 \sin 2 \varphi k_{20,2}^{12} P_{20}^{2}(\cos \theta) \cdot \frac{U d S}{a^{2}} .
$$


(a)
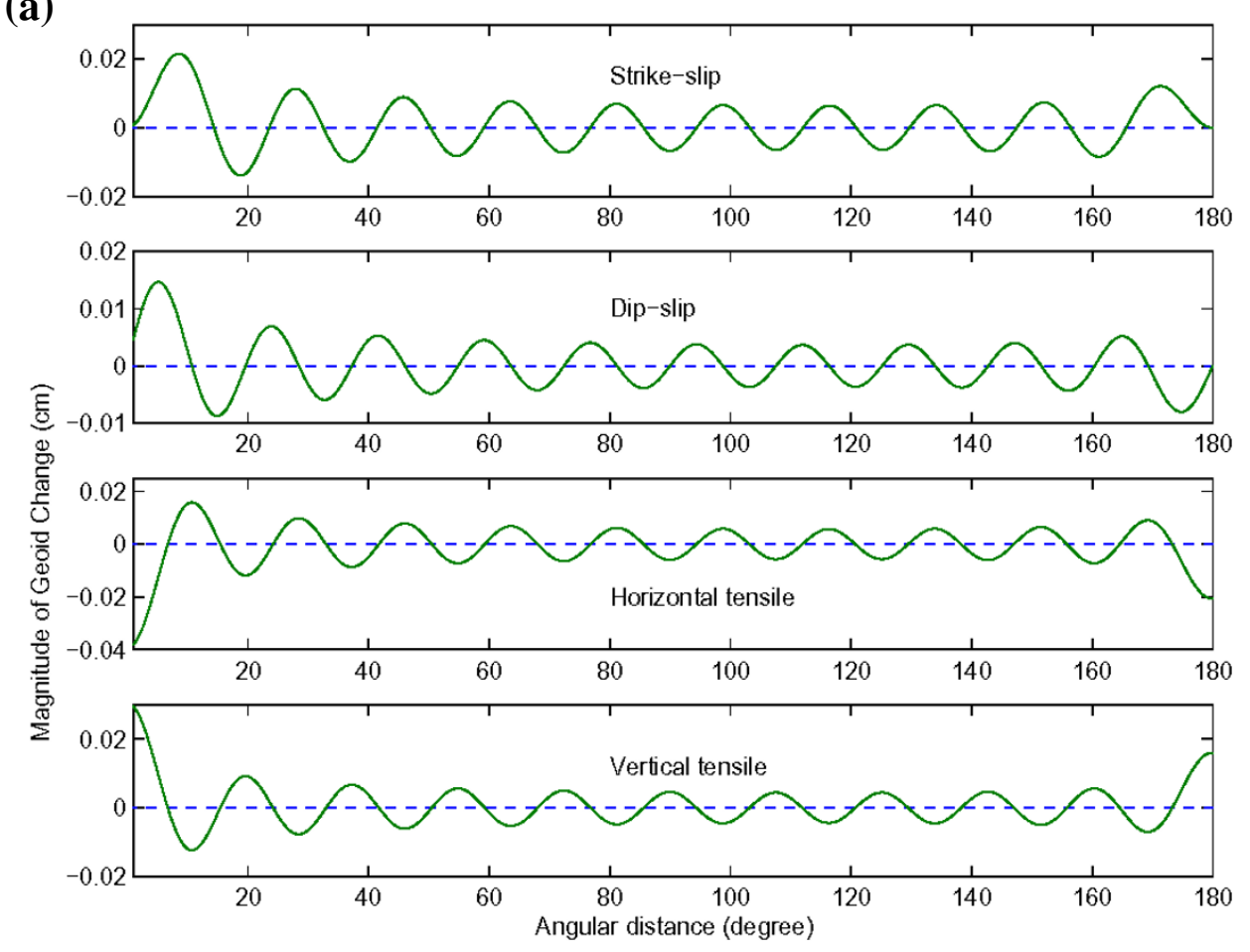

(b)
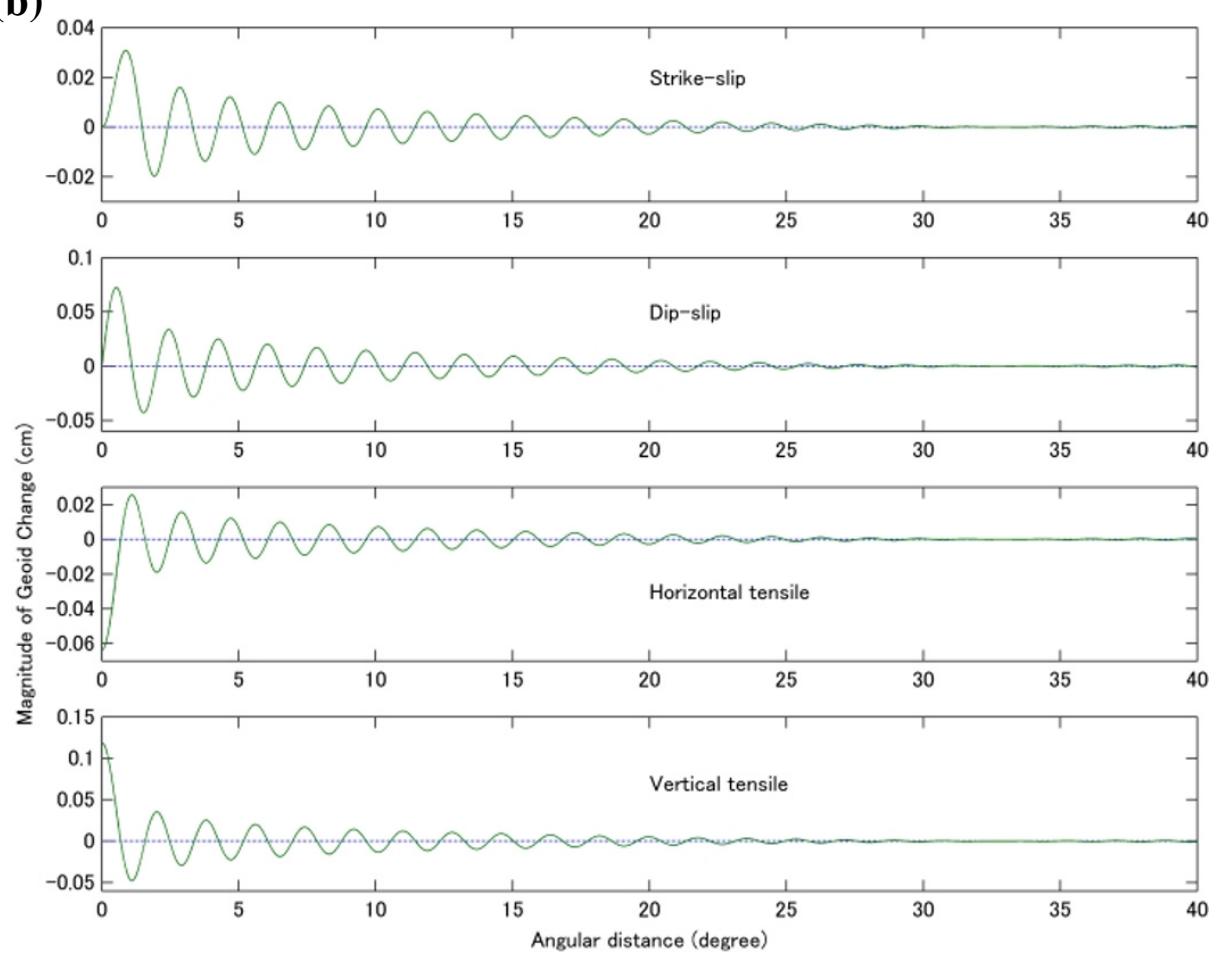

Fig. 4. Co-seismic geoid changes of four independent solutions for spherical harmonic degrees 20 (a) and 200 (b), caused by a dislocation at depth of 32 $\mathrm{km}$. The dislocation factor is $U d S / a^{2}=2.956 \mathrm{~cm}$, equivalent to the size of the Alaska earthquake $(1964,9.2)$.

Similar formulas can be written for other components. Using the seismic parameters presented above, we calculate soseismic geoid and gravity changes for the four independent solutions considering only individual wavelengths of 1000 $\mathrm{km}$ and $100 \mathrm{~km}$, which correspond to harmonic degrees of $n=20$ and $n=200$, respectively. Results are plotted in Figs. 4(a) and 4(b), respectively. They show that the co- seismic geoid height changes reach $0.2-1.0 \mathrm{~mm}$. The results for the low degree of $n=20$ indicate that co-seismic geoid changes distribute over the whole earth surface with almost equal magnitude; whereas results of the high harmonic degree of $n=200$ have large magnitude in near field and decay fast as the epicentral distance increases. However, both low and high degrees have equal contribution in magnitude. The 
(a)
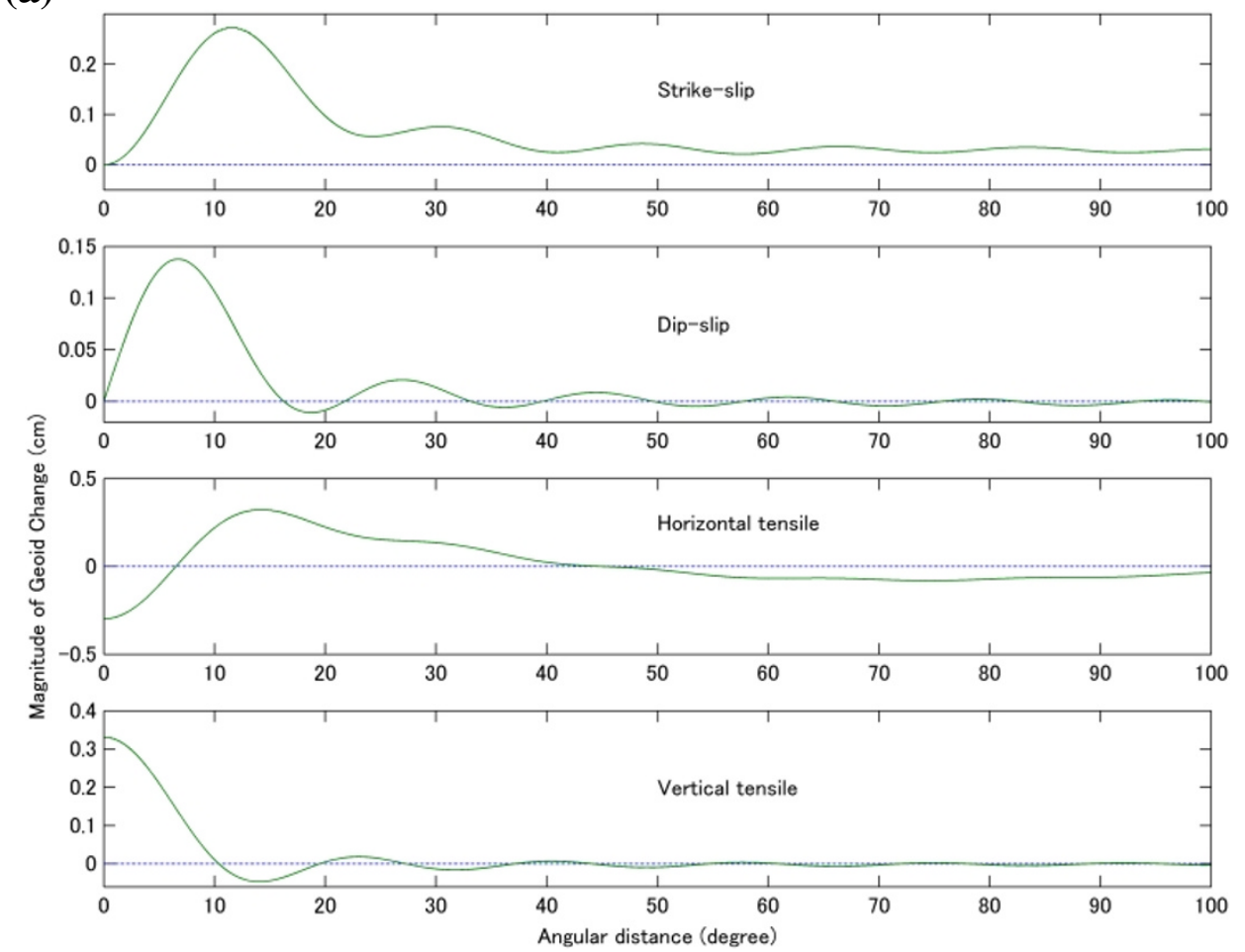

(b)
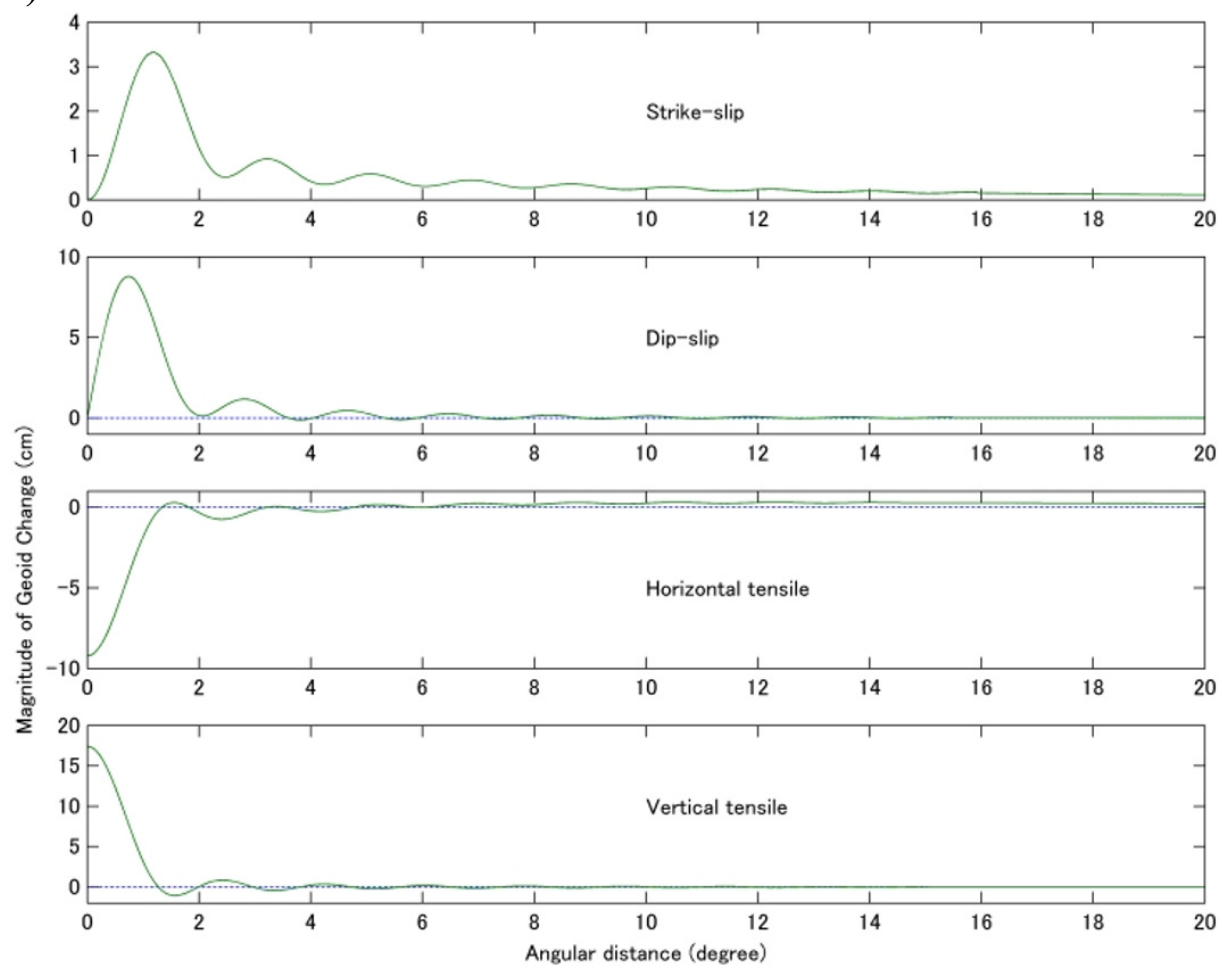

Fig. 5. Co-seismic geoid changes of four independent solutions for the first 20 (a) and 200 (b) harmonic degrees caused by the same source as above.

co-seismic geoid changes are two orders larger than the precision of GRACE (ESA, 1999) and are certainly detectable, especially for the low harmonic degrees. Note that conclusions obtained in this section should be identical to those in Section 3 because the two sections discuss the same problem from two aspects.

\section{Truncated Co-seismic Geoid and Gravity Changes}

The dislocation theory for a spherical earth (e.g., Sun and Okubo, 1993) can be used to calculate a total co-seismic geoid or gravity change by summation of the dislocation Love numbers over all harmonic degrees. On the other 
hand, Sections 3 and 4 above explain how to calculate coseismic deformations for each individual harmonic. Sometimes, however, we may need to consider the cumulative coseismic deformations because the gravity satellite missions cannot detect whole components of degree $n$ as a result of its spatial resolution limit. For this purpose, we should calculate co-seismic deformation with a truncation, so that truncated co-seismic deformations can be calculated and be compared to the gravity missions. Therefore, we define here a concept of truncated co-seismic geoid and gravity changes. That is, co-seismic deformations are calculated only for an interested degree band, i.e., the summations in Eqs (1) and (2) are performed from $N_{1}$ to $N_{2}$ to yield

$$
\begin{gathered}
\zeta^{i j}(a, \theta, \varphi)=\sum_{n=N_{1}}^{n=N_{2}} \sum_{m} k_{n m}^{i j} Y_{n}^{m}(\theta, \varphi) \cdot v_{i} n_{j} \frac{U d S}{a^{2}} \\
\delta g^{i j}(a, \theta, \varphi)=\sum_{n=N_{1}}^{n=N_{2}} \sum_{m}(n+1) k_{n m}^{i j} Y_{n}^{m}(\theta, \varphi) \cdot v_{i} n_{j} \frac{g_{0} U d S}{a^{3}} .
\end{gathered}
$$

As an example, the explicit expressions of the truncated coseismic geoid and gravity changes for the strike-slip source can be written as

$$
\begin{gathered}
\zeta^{12}(a, \theta, \varphi)=2 \sin 2 \varphi \sum_{n=N_{1}}^{n=N_{2}} k_{n 2}^{12} P_{n}^{2}(\cos \theta) \cdot \frac{U d S}{a^{2}} \\
\delta g^{12}(a, \theta, \varphi)=2 \sin 2 \varphi \sum_{n=N_{1}}^{n=N_{2}}(n+1) k_{n 2}^{12} P_{n}^{2}(\cos \theta) \cdot \frac{g_{0} U d S}{a^{3}}
\end{gathered}
$$

Using Eqs. (9) and (10), we can calculate the co-seismic geoid changes for the first 20 and 200 harmonic degrees: the summations are made from $N_{1}=2$ to $N_{2}=20$ and $N_{2}=200$, respectively. Results are plotted in Figs. 5(a) and 5(b), respectively. We found that the co-seismic geoid changes are as large as $2-200 \mathrm{~mm}$. It can also be seen that the more harmonic degrees are included, the larger the coseismic geoid changes. As indicated in the above sections, tensile source deformations are larger than those of the shear sources. On the other hand, as expected, the summation results for $N_{2}=20$ show low frequency behavior, whereas deformations for summation to $N_{2}=200$ have high frequency variation. We have similar results for co-seismic gravity changes. The results show that they vary from several hundred microgals to $1000 \mu$ gals.

Note that the above results in Figs. 4 and 5 represent coseismic deformations of a point source (dislocation). In practice, if the fault size is very large or compatible with the distance between the source and satellite, the geometrical shape of the fault should be considered. If the fault size is sufficiently small in comparison to the distance from satellite to the earth surface, a point source is sufficient. On the other hand, source depth is another factor affecting the magnitude of deformations. However, compared to the fault size, the effect of source depth is considered to be relatively small because co-seismic deformation, especially the geoid change, is not so sensitive to depth (Sun and Okubo, 1998). Note that the finite size of the fault might be important for the GRACE follow-on mission. To reduce contamination from hydrology, oceanography, and other factors, we should probably address as small a region as possible because relatively small follow-on errors will permit us to examine those very small regions: small enough that the fault size plane extent for a large event could be important.

An integration of a point source over fault plane is required to compute accurate co-seismic deformations by a limited fault size. However, this study is intended to observe magnitude of co-seismic deformations. A rough approximation is acceptable. Therefore instead of integration, we compute the co-seismic deformations using a moving smoothness over epicentral distance on the above results obtained for a point source. This is equivalent to considering a line source inside the earth. Our calculation adopts $600-\mathrm{km}$ smoothness (the same size as the fault length of the Alaska earthquake, 1964). This approximation is considered good enough for the research. Results of the co-seismic geoid changes for an approximated fault plane are calculated and plotted in Figs. 6(a) and 6(b). Figure 6 shows that results change only slightly compared to those of the point source. This is understandable because the truncated coseismic geoid changes represented in Figs. 4 and 5 include wavelengths over $1000 \mathrm{~km}$ and $100 \mathrm{~km}$, respectively, which are much longer than the fault length. Actually, a source with a limited fault length can still be treated as a point source for a low degree or long wavelength. Similarly, gravity changes of the first 20 and 2000 degrees for a limited fault reach about 2-300 $\mu \mathrm{gal}$ and 400-5000 $\mu \mathrm{gal}$, respectively.

\section{Case Study-Alaska Earthquake (M=7.9, 2002)}

As a case study, we address the Alaska earthquake $(M=7.9)$ which occurred on Nov. 3, 2002. This earthquake was accompanied by a vertical strike-slip movement at a depth of $15 \mathrm{~km}$ with a fault size of $200 \mathrm{~km} \times 30 \mathrm{~km}$. The average movement (dislocation) is about $4.3 \mathrm{~m}$ (Fig. 7).

According to fault size, the dislocation factors for geoid and gravity changes can be obtained as: $U d S / a^{2}=0.64$ $\mathrm{mm}$ and $g_{0} U d S / a^{3}=0.098 \mu g a$. Then, inserting the factors and dislocation Love numbers, which are numerically calculated for a vertical strike-slip source at a depth of $15 \mathrm{~km}$ in the 1066A earth model, into Eqs. (6) and (7), we calculate the corresponding co-seismic geoid and gravity changes for harmonic degrees of 2, 10 and 20, respectively. Results of the geoid changes are plotted in Fig. 8. It is seen from Fig. 8 that the geoid change for the harmonic degree of $n=20$ distributes over the whole earth surface with one half-wavelength, as expected. In contrast, results for $n=10$ and $n=20$ appear to have relative high frequency variations. It also shows that these geoid changes for different harmonic degrees have almost identical contributions in magnitude. The maximum geoid change is about 0.008 $\mathrm{mm}$ for $n=2,0.006 \mathrm{~mm}$ for $n=10$, and $0.005 \mathrm{~mm}$ for $n=20$. Comparing these results with the anticipated geoid height errors derived from GRACE data (Table 2), we found that the geoid changes caused by the 2002 Alaska earthquake $(m=7.9)$ are approximately the same level as the error, but slightly smaller. That is, these geoid changes are difficult to detect by GRACE.

On the other hand, co-seismic gravity changes caused by this earthquake reach $0.0013 \mu$ gals for $n=2,0.0008 \mu$ gals 
(a)
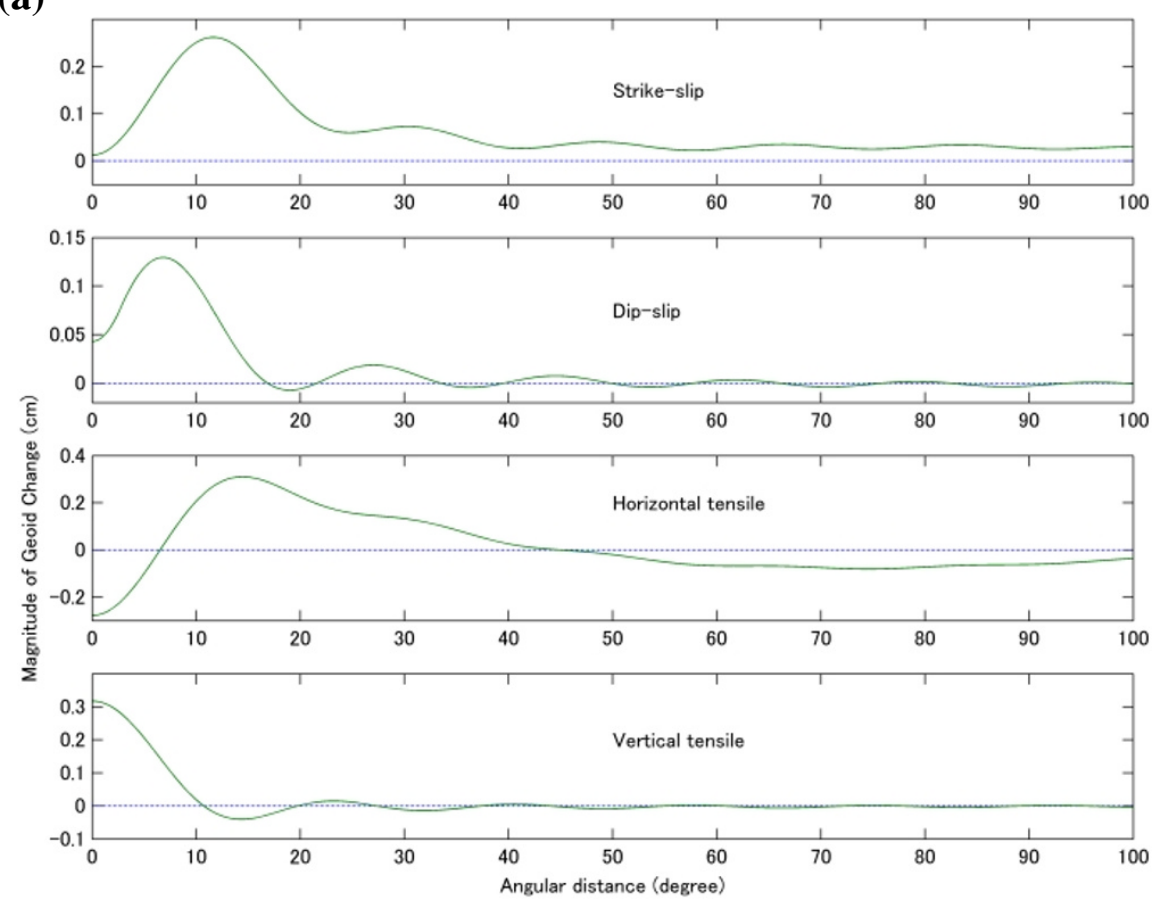

(b)
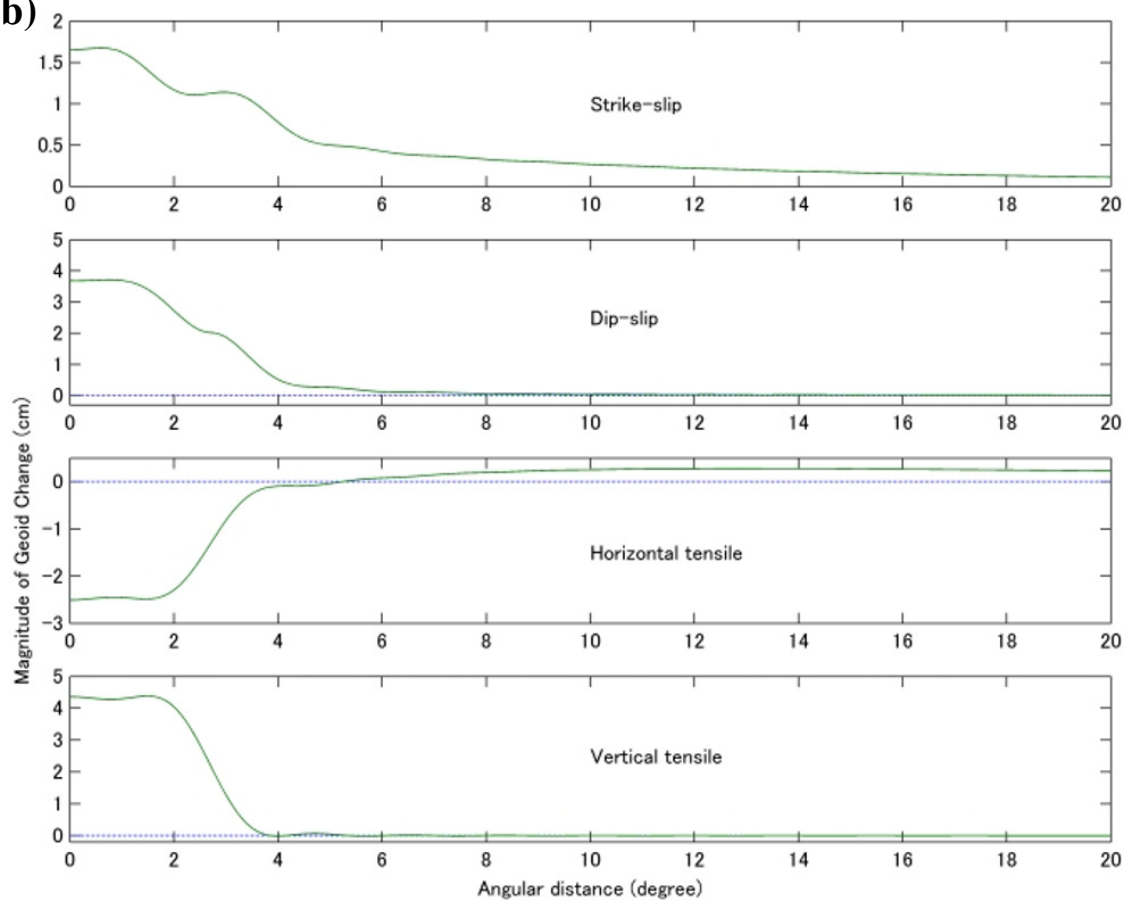

Fig. 6. Co-seismic geoid changes of four independent solutions for the first 20 (a) and 200 (b) harmonic degrees, by $600 \mathrm{~km}$ moving smoothed.

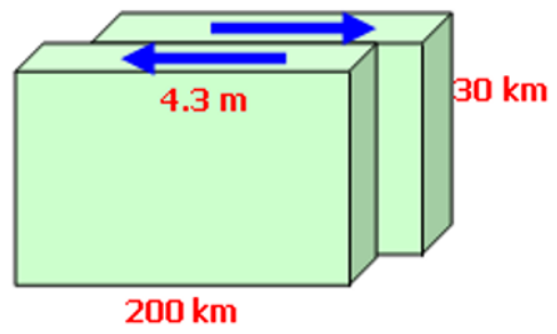

Fig. 7. Fault parameters of the 2002 Alaska earthquake $(m=7.9)$. for $n=10$, and $n=20$. Behaviors of gravity changes are fundamentally identical to those of the geoid changes. A comparison of results with the expected gravity anomaly errors (ESA, 1999) shows that these changes are at the same level as the errors, but that they are slightly larger (see the result of $n=20$ ) and not remarkable.

It should be pointed out that seismic gravity changes are difficult to distinguish in practice because of the complication of the gravity field. Ideally, to distinguish co-seismic geoid and gravity changes, the gravity field should be ob- 

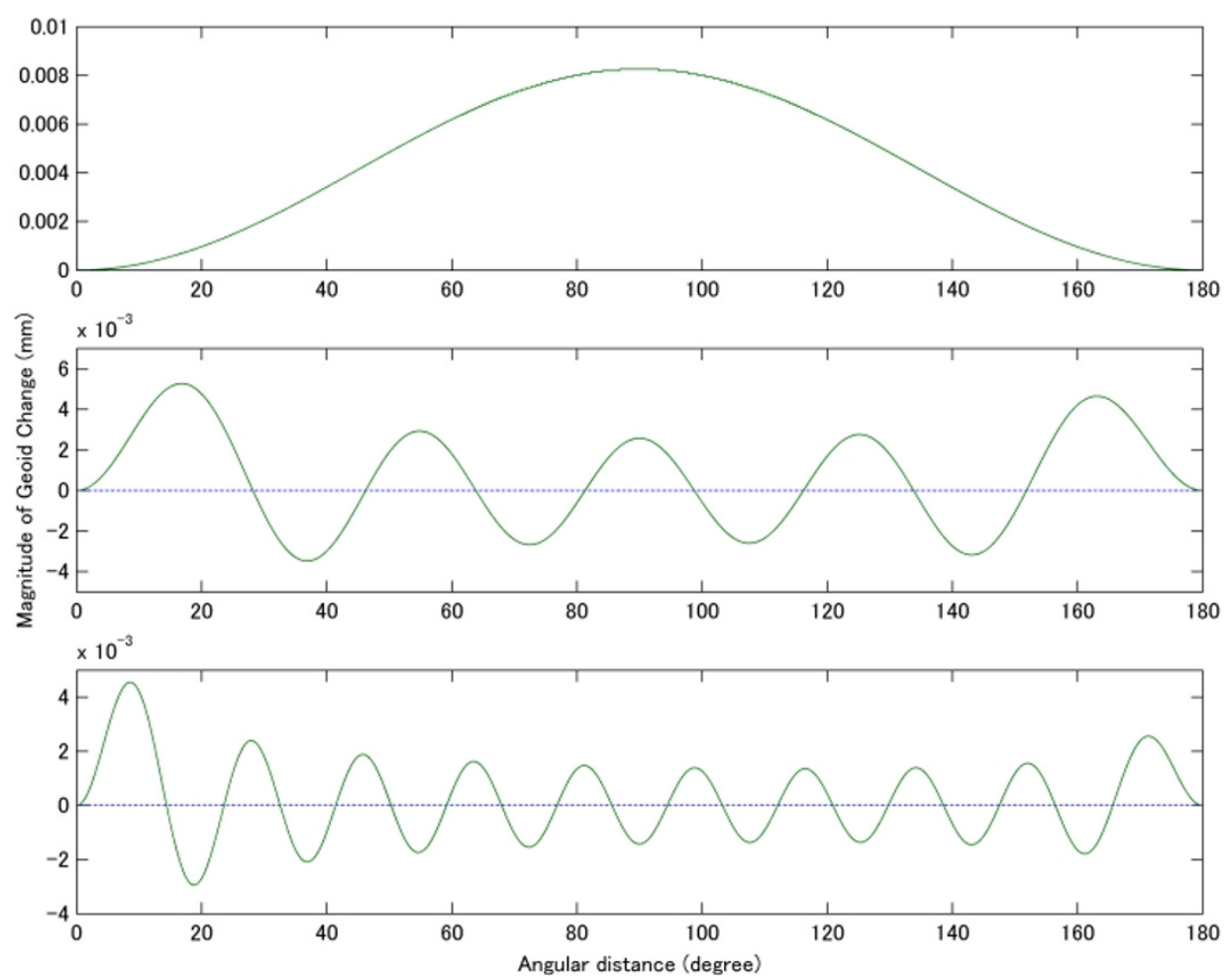

Fig. 8. Co-seismic geoid changes caused by the 2002 Alaska earthquake $(m=7.9)$. From the top to the bottom are results for spherical harmonic degrees of 2,10 and 20 , respectively.

served just before and after the seismic event. In this case, all the other long temporal effects on gravity change should be relative small and can be neglected. In practice, however, GRACE provides us with a complete gravity observation in a one month time interval. During that one month, the earth undergoes many geophysical changes that engender temporal gravity changes. In other words, the temporal gravity variations are expected to be composed of many physical effects, such as tidal changes, atmospheric changes, rain or snow fall, and so on. On the other hand, in some time scale, the other temporal gravity variations are identical or even larger than the co-seismic deformations. Therefore, all of these gravity changes should be well modeled or observed before the co-seismic gravity changes can be detected.

The above results and discussions imply that co-seismic geoid and gravity changes are almost impossible to detect by GRACE. However, for an earthquake with a magnitude bigger than $m=7.9$, such as $m>8.0$ as shown in Table 3, co-seismic geoid and gravity changes are anticipated to be detectable by GRACE. On a hopeful note, because the forthcoming GRACE follow-on gravity mission is expected to be better than GRACE by more than two orders in accuracy, geoid and gravity changes caused even by the 2002 Alaska earthquake may be detectable from space by projects such as the GRACE follow-on mission.

\section{Discussion and Final Remarks}

In this paper, the conventional quasi-static dislocation theory for a spherical earth model, e.g., the theory of Sun and Okubo (1993) is straightforwardly used to derive expressions for computing co-seismic deformations for each individual or group harmonic degree. Numerical investigation is carried out to observe whether or not co-seismic geoid and gravity changes are detectable by satellite gravity missions. A seismic source equivalent to the fault size of the great Alaska earthquake $\left(1964, m_{w}=9.2\right)$ is adopted. The results of co-seismic deformations indicate that both the gravity and geoid changes are about two orders larger than the precision of GRACE. The minimum earthquakes to be detected by GRACE and GRACE follow-on are correspondingly derived. The conclusion is that co-seismic deformations for an earthquake with a seismic magnitude of $m=7.5$ (for tensile sources) and $m=9.0$ (for shear sources) are expected to be detectable by GRACE, which confirms the same conclusion as Gross and Chao (2001) and our previous study (Sun and Okubo, 2004). It should be pointed out that the above conclusion is derived based on the four independent seismic sources, i.e., the vertical strike-slip, vertical dip-slip, horizontal extension on a vertical fault and vertical extension on a horizontal fault. For a real case, the co-seismic deformations are a combination of the four sources, where the dip angle and slip angle play an important role. It implies that real co-seismic deformations vary depends on the strike/dip angle although for the same seismic magnitude. Then, a case study on the 2002 Alaska earthquake ( $m=7.9$ ) was made. Results show that the co-seismic geoid and gravity changes are just at the error level of GRACE. It seems difficult to be detected by GRACE. Finally, an overview is given to discuss the relation of this work and the previous studies. GRACE delivers the difference of two monthly gravity maps, which is very small everywhere but showing a relative large distribution pattern in near field for an earthquake. The spatialdistributed difference can be expressed as either individual harmonic degree or total effect. In this research we are inter- 
ested in the spatial distribution of each individual or group spherical harmonic degree since the dislocation theory (Sun and Okubo, 1993) can be straightforwardly used for this purpose, not the total sum of them (since the high degree parts can not be observed from space). Note that the dislocation theory for a half-space does not apply for this purpose. What we do in this research is to consider the spatial distribution of each harmonic degree, and then the results are used to compare with the GRACE sensitivity. Actually, this is a parallel way to Gross and Chao's (2001) and Sun and Okubo's (2004) approaches. Gross and Chao's (2001) approach deals with the problem in spectrum domain using normal mode technique. Sun and Okubo's (2004) approach treats the same problem also in the spectrum domain but taking the dislocation Love numbers into account, so that the detectability of GRACE can be investigated for different source types. However, the current approach discusses the problem in spatial domain for each spherical harmonic degree. Using the spatial domain is to take the advantage of the dislocation theory. The formulation and results presented in this paper have the following implications: 1) the results independently lead to the same conclusion as the two previous studies. 2) This research opens a way to study great earthquakes in the subduction zones, where usually lack geodetic observations on the seaward. 3) It can be used to study deformations caused by slow earthquakes which can not be determined by the conventional seismic method. Therefore, the theory and results in this paper are expected to have wider applications in the future.

Acknowledgments. The authors are grateful for comments by B. Vermeersen, B. F. Chao, R. Gross and an anonymous reviewer. This research was supported financially by Japan Society for the Promotion of Science research grants (C16540377) and "Basic design and feasibility studies for the future missions for monitoring Earth's environment".

\section{References}

Chao, B. F., Geodesy is not just for static measurements any more, Eos, Transactions, American Geophysical Union, 84, 145-156, 2003.

Dziewonski, A. M. and D. L. Anderson, Preliminary Reference Earth Model, Phys. Earth Planet. Inter., 25, 297-356, 1981.

ESA, Gravity field and steady-state ocean circulation mission, Reports for mission selection: The four candidates earth explorer core missions, SP1233 (1), 1999.

Gilbert, F. and A. M. Dziewonski, An application of normal mode theory to the retrieval of structural parameters and source mechanisms from seismic spectra, Phil. Trans. R. Soc. London, A 278, 187-269, 1975.

Gross, R. S. and B. F. Chao, The gravitational signature of earthquakes, in Gravity, Geoid, and Geodynamics 2000, edited by M. G. Sideris, pp. 205-210, IAG Symposia Vol. 123, Springer-Verlag, New York, 2001.

National Research Council, NAS, Satellite Gravity and the Geosphere, edited J. O. Dickey, Washington, D.C., 1997.

Pollitz, F. F., Gravitational-viscoelastic postseismic relaxation on a layered spherical Earth, J. Geophys. Res., 102, 17921-17941, 1997.

Savage, J. C. and L. M. Hastie, Surface deformation associated with dip-slip faulting, J. Geophys. Res., 71, 4897-4904, 1966.

Sun, W. and S. Okubo, Surface potential and gravity changes due to internal dislocations in a spherical earth-I. Theory for a point dislocation, Geophys. J. Int., 114, 569-592, 1993.

Sun, W. and S. Okubo, Surface potential and gravity changes due to internal dislocations in a spherical earth-II. Application to a finite fault, Geophys. J. Int., 132, 79-88, 1998.

Sun, W. and S. Okubo, Co-seismic Deformations Detectable by Satellite Gravity Missions-a Case Study of Alaska $(1964,2002)$ and Hokkaido (2003) Earthquakes in Spectral Domain, J. Geophys. R., 109(B4), B04405, doi:10.1029/2003JB002554, 2004.

Sun, W., S. Okubo, and P. Vanicek, Global displacement caused by dislocations in a realistic earth model, J. Geophys. Res., 101, 8561-8577, 1996.

Wang, H., Surface vertical displacements, potential perturbations and gravity changes of viscoelastic earth model induced by internal point dislocations, Geophys J. Int., 137, 429-440, 1999.

Watkins, M. M., W. M. Folkner, B. F. Chao, and B. D. Tapley, The NASA EX-5 Mission: A laser interferometer follow-on to GRACE, IAG Symp. GGG2000, Banff, July, 2000.

W. Sun (e-mail: sunw@eri.u-tokyo.ac.jp) and S. Okubo 